\title{
Difesa fitosanitaria e sicurezza alimentare
}

\author{
Annamaria Vercesi, ${ }^{1}$ Piero Cravedi \\ ${ }^{1}$ Dipartimento di Produzione Vegetale, Università di Milano; ${ }^{2}$ Istituto di Entomologia e Patologia \\ Vegetale, Università Cattolica del sacro Cuore di Piacenza, Italy
}

\begin{abstract}
Pest management is essential to limit the damages due to the biotic agents of crop losses and obtain adequate yield characterized by high quality standards. Agricultural practice, based on the cultivation of several genetically uniform 0 very similar crops in contiguous fields, often provides ideal conditions for severe outbreaks of diseases/infestations due to pathogens and insects able to attack the host plant grown in a particular area. Pathogens and insects can cause serious losses, varying from 10 to $40 \%$ of the global crop production. Resistant hosts and plant protection products are the control tools most commonly utilized in order to gain effective reduction of crop damages. Since plant protection products can have harmful effects on human health, non-target organisms and environment, their registration and use are regulated in the European Community according to the precautionary principle. Moreover, the European Community countries will implement integrated protection management measures on all cultivated crops starting from 1st January 2014 and use risk indicators in order to define more rational pest management strategies.
\end{abstract}

\section{Riassunto}

La difesa fitosanitaria è indispensabile per limitare i danni dovuti alle avversità biotiche delle piante coltivate e ottenere produzioni soddisfa-

\footnotetext{
Correspondence: Annamaria Vercesi, Dipartimento di Produzione Vegetale, sez. Patologia Vegetale, via Celoria 2, 20133 Milano, Italy.

Tel. +39.02.50316776 - Fax: +39.02.50316781.

E-mail: annamaria.vercesi@unimi.it
}

Key words: pathogens, insects, plant protection products, integrated pest management.

Parole chiave: patogeni, parassiti, agrofarmaci, protezione integrata

Received for publication: 11 April 2011.

Accepted for publication: 30 May 2011.

Società rappresentata nell'ambito AISSA: Associazione Italiana Protezione delle Piante - AIPP.

(C) Copyright A. Vercesi and P. Cravedi, 2011

Under no circumstances figures can be used without prior written consent of the copyright owner.

Licensee PAGEPress, Italy

Italian Journal of Agronomy 2011; 6(s2):e5

doi:10.4081/ija.2011.6.s2.e5

This work is licensed under a Creative Commons Attribution NonCommercial 3.0 License (CC BY-NC 3.0). centi in termini qualitativi e quantitativi. La pratica dell'agricoltura, mettendo a disposizione di patogeni e parassiti ospiti caratterizzati da uniformità genetica riuniti in spazi ristretti, determina un incremento dell'incidenza di malattie/infestazioni. I patogeni ed i parassiti sono in grado di provocare danni molto gravi che secondo le stime disponibili possono variare tra il 10 ed il $40 \%$ dell'intera produzione mondiale. I mezzi maggiormente utilizzati per la protezione delle piante sono di natura genetica e chimica. L'uso di mezzi chimici, la cui applicazione può comportare rischi per la salute dell'uomo, per gli organismi non bersaglio e per l'ambiente, è regolamentata in Europa da disposizioni legislative che si ispirano al principio di precauzione e che puntano a conseguire una limitazione del danno razionalizzando al meglio le strategie di intervento mediante l'utilizzazione di indicatori di rischio.

Il ruolo della difesa sanitaria nel garantire la sicurezza alimentare consiste nell'individuare mezzi e strategie efficaci per contenere la riduzione della produzione di derrate alimentari causata dalle avversità delle piante coltivate e spontanee, i cui agenti biotici vengono definiti nel loro complesso pests dagli studiosi di lingua inglese. I pest includono organismi patogeni quali funghi e batteri, virus, viroidi, fitoplasmi, animali, in stragrande maggioranza insetti, ed erbe infestanti. Benché gli agenti biotici di avversità possano compromettere annualmente parti rilevanti dell'intera produzione agricola mondiale, l'impatto di parassiti e patogeni sulla sicurezza alimentare viene spesso trascurato, diversamente da quanto accade non solo per la salute umana, ma anche per quella animale (Flood, 2010). Ciò non di meno sono noti esempi di carestie provocate da organismi fitopatogeni che hanno avuto conseguenze molto importanti anche da un punto di vista sociale: valga per tutti l'esempio della peronospora della patata, causata da Phytophthora infestans (Mont.) De Bary, che venne introdotta dal Messico in Europa nel 1844 e negli anni successivi determinò epidemie gravissime, in particolare in Irlanda (Fry, 2008). Milioni di irlandesi, per i quali la patata costituiva la principale fonte di sostentamento, morirono o emigrarono, principalmente verso gli Stati Uniti. La popolazione dell'isola passò dai 6 milioni rilevati nel 1845 ai 4 milioni di abitanti accertati nel 1850. La potenzialità distruttiva degli organismi fitopatogeni, più volte rilevata nel passato, costituisce ancor oggi motivo di allarme. Nel 1999 è stata segnalata su grano in Uganda una nuova razza di Puccinia graminis f. sp. tritici Erikss. e Henning, denominata Ug99 (Pretorius et al., 1999) che presenta alcune allarmanti caratteristiche: Ug99 è infatti in grado di superare la resistenza conferita sia dal gene Sr31, originariamente presente in segale ed attualmente contenuto in moltissime varietà di frumento diffuse in India, Cina, Europa ed America latina, sia dal gene Sr38, proveniente da Triticum ventricosum L., introiettato in varietà coltivate in Europa ed Australia. Questi due geni di resistenza fanno parte del patrimonio genetico di molte varietà di frumento messe a punto durante la Rivoluzione Verde, che hanno grandemente contribuito all'incremento della produzione mondiale di questo cereale (Singh et al., 2006). Ug99 è estremamente aggressiva e, come tutte le ruggini, può essere trasportata a notevole distanza dai venti dominanti. In considerazione della direzione di tali venti sono state individuate due vie principali di diffusione, la Route $A$, che passa attraverso la penisola arabica e prosegue verso 
l'Iran, il Pakistan e l'India, e la Route $B$, che arriva direttamente attraverso l'Oceano Indiano dall'Africa al subcontinente indiano. Dagli ultimi rilevamenti, il percorso di diffusione di Ug99 coincide con la Route A: danni di notevole entità si sono verificati oltre che in Africa anche nello Yemen nel 2003 e in Iran tra il 2007 ed il 2008. La comunità internazionale sta seguendo attentamente l'evoluzione della situazione, cercando di correre ai ripari, anche se non sembra facile mettere a punto varietà dotate di resistenza nei confronti di Ug99, nonostante siano state identificate fonti di resistenza (Njau et al., 2010). In campo entomologico si può citare il caso devastante dell'arrivo dal Nord America della fillossera della vite, che comportò la ricostituzione della viticoltura europea, ricordando che l'arrivo di nuove specie di insetti dannosi è continua. Un caso recente riguarda l'introduzione del Lepidottero Tuta absoluta Povolny dannoso al pomodoro.

D'altro canto il passaggio da un ecosistema naturale ad un agroecosistema comporta, come conseguenza inevitabile, l'inasprirsi di problemi legati al verificarsi di avversità biotiche: la pratica agricola mette a disposizione degli agenti di tali avversità un gran numero di piante geneticamente uniformi, in continuità spaziale e temporale tra di loro. Da un punto di vista agroecologico, le colture agricole sono caratterizzate da quattro fattori fondamentali: la produttività, la stabilità intesa come costanza della produzione a dispetto delle normali variazioni delle condizioni ambientali, la resilienza, vale a dire la capacità di rimediare ad effetti negativi indotti da condizioni sfavorevoli e l'equità, in altre parole la ripartizione del prodotto tra i beneficiari (Conway, 2007). Una variazione in uno di questi fattori causa un corrispondente cambiamento nei rimanenti: in particolare l'incremento di produttività si ripercuote in genere negativamente su stabilità e resilienza. La Rivoluzione Verde ha comportato l'introduzione su vasta scala di varietà di cereali caratterizzati da un'elevata produttività, ma è stata anche accompagnata da un incremento nell'utilizzo di fertilizzanti, acqua e, a causa dell'aumentata incidenza di malattie/infestazioni, di prodotti fitosanitari (Evenson e Gollin, 2003). Le nuove varietà prodotte dalla rivoluzione verde erano infatti state messe a punto in ambienti caratterizzati da ampia disponibilità di acqua, fertilizzanti e nuovi agrofarmaci dotati di notevole efficacia, disponibilità non sempre riscontrabile in altri agroecosistemi. Si parla attualmente di una seconda 0 addirittura di una terza Rivoluzione Verde, basata su piante adattate a situazioni di ridotta fertilità e disponibilità idrica, maggiormente resistenti alle avversità di origine biotica e sulla coesistenza tra specie/varietà diverse (Beddington, 2010).

Dati quantitativi affidabili sull'impatto che le avversità biotiche delle piante hanno sulla produzione sono molto carenti per una serie di ragioni: in primo luogo non viene effettuata una raccolta sistematica di informazioni a livello nazionale ed internazionale e secondariamente molti dei dati attualmente disponibili sono in realtà delle stime basate sulla relazione esistente tra l'incidenza dell'alterazione provocata dagli agenti biotici o dalla loro presenza da un lato e la perdita di produzione dall'altro (Oerke, 2006). Il danno può essere valutato in termini di riduzione pura e semplice della produzione 0 con riferimento alla perdita finanziaria che ne deriva. Quest'ultima valutazione è soggetta a molte variazioni dovute all'oscillazione dei prezzi che spesso non dipendono solo da decrementi nella produzione, ma da altri fattori quali speculazioni di vario tipo. A questo si aggiunga che l'incidenza delle avversità cambia notevolmente in funzione della coltura considerata e, sulla stessa pianta coltivata, di anno in anno e nelle varie zone di coltivazione.

I dati più attendibili riguardano i paesi industrializzati, mentre le informazioni riguardanti i paesi in via di sviluppo, dove le condizioni climatiche, soprattutto nelle aree tropicali e subtropicali, sono decisamente favorevoli ad una proliferazione di batteri e funghi patogeni e di insetti, sono spesso carenti 0 addirittura assenti. Le stime relative alle perdite dovute ad agenti di avversità biotiche sono molto controverse: si va dal $10 \%$ al $40 \%$ secondo la FAO, mentre secondo altre fonti almeno il $30 \%$ della produzione va perduto a causa di patogeni e parassiti (Strange e Scott, 2005; Bentley et al., 2009; Godfray et al., 2010). Le perdite possono avvenire sia in campo sia durante l'immagazzinamento: mentre tutti gli agenti di avversità biotiche sono in grado di danneggiare le colture in campo, in postraccolta sono soprattutto i funghi, gli insetti ed i roditori a fare la parte del leone, causando perdite che si aggirano secondo le stime più attendibili sul $10 \%$ della produzione ottenibile. L'entità dei danni di cui sono responsabili gli agenti delle avversità biotiche sulle varie colture sono molto variabili: le malerbe determinano le perdite più consistenti, seguite dagli insetti che tuttavia su grano e patata vengono superati da funghi e virus (Oerke, 2006). Va sottolineato inoltre che le stime effettuate prendono raramente in considerazione il deprezzamento delle derrate indotto dall'accumulo di micotossine, metaboliti tossici per l'uomo e gli animali, prodotte da alcune specie di funghi fitopatogeni.

L'applicazione di strategie di difesa riduce in modo molto significativo i danni causati dalle malerbe, mentre risulta meno efficace nei confronti di patogeni ed insetti. I mezzi che vengono utilizzati per ridurre i danni dovuti ad avversità biotiche sono meccanici, fisici, genetici, agronomici, biologici e chimici. I mezzi meccanici, che comportano l'eliminazione manuale o con opportuni attrezzi dell'agente di danno, vengono utilizzati solo nei confronti delle malerbe, mentre tra i mezzi fisici trova impiego in campo fitoiatrico il calore che, se elevato, devitalizza i propaguli di funghi, batteri, insetti e malerbe, e, se ridotto, rallenta lo sviluppo di patogeni e parassiti durante il post raccolta. I mezzi genetici, utilizzati in gran parte nelle colture annuali, sono rappresentati dall'introgressione di geni che conferiscono generalmente resistenza nei confronti di una razza del patogeno che la pianta riconosce come altro da sé e provvede ad escludere. Più utilizzati nel caso di piante arboree sono le resistenze di tipo quantitativo che sono meno selettive, ma più durevoli. Mezzi genetici sono anche utilizzati per la messa a punto di Organismi Geneticamente Modificati, sui quali sussistono molte perplessità anche dal punto di vista fitoiatrico: l'inserimento del gene Bt ha realmente contribuito ad una razionalizzazione della difesa. Infine i mezzi agronomici mirano a ridurre le condizioni favorevoli all'insediamento e/o sviluppo degli agenti biotici delle avversità delle piante coltivate e, benché non risolutivi, possono contribuire in modo efficace alla protezione delle colture. I mezzi biologici di difesa sono considerati, soprattutto dai non addetti ai lavori, gli strumenti di difesa delle piante coltivate a minor impatto ambientale: se parecchi successi sono stati registrati da questo punto di vista in campo entomologico, in campo fitopatologico le cose non stanno esattamente così; ad esclusione di alcuni casi molto particolari, a decenni di distanza dall'inizio delle ricerche in quest'ambito, non esiste alcun esempio di utilizzazione su vasta scala di mezzi biologici di difesa su colture di rilevante importanza.

In modo completamente diverso vengono percepiti i prodotti fitosanitari, gli agrofarmaci comunque li si voglia chiamare tranne che pestici$\mathrm{di}$, che sono infatti considerati dall'opinione pubblica i principali responsabili di inquinamento delle derrate e dell'ambiente con conseguenze deleterie sulla salute del consumatore e su quelli che in gergo tecnico vengono chiamati organismi non bersaglio. Gli agrofarmaci balzano prepotentemente alla ribalta nel secondo dopoguerra, ma gli sforzi per mettere a punto sostanze impiegabili a scopo fitoiatrico in campo agricolo iniziano alla fine della prima guerra mondiale, quando le industrie chimiche del settore perdono i proventi derivanti dalle produzioni connesse con l'attività bellica (Russell, 2006). Tisdale e Williams (1934) che lavorano per Du Pont registrano nel 1934 un brevetto riguardante i dimetilditiocarbammati, una classe di composti chimici che mostra una notevolissima attività antifungina e nel 1940 viene posto in commercio il primo fungicida organico di sintesi, il thiram. Dal secondo dopoguerra ad oggi, ma soprattutto nella seconda metà del XX secolo vengono introdotti sul mercato agrofarmaci caratterizzati da un'elevata efficacia, dosi d'impiego ridotte e, dal 1969 in avanti, fungicidi in grado di penetrare all'interno della pianta sulla quale vengono applicati con conseguente redistribuzione interna, nessun rischio di dilavamento, efficacia post-infezionale. L'affermarsi degli agrofarmaci è stato spesso accompagnato da un uso disinvolto delle sostanze attive disponibili, che specialmente al loro com- 
parire sono state ritenute la panacea di tutti i mali. Gli effetti negativi degli agrofarmaci sulla salute dell'uomo e sull'ambiente cominciarono ad essere considerati a partire dalla metà degli anni 50 del secolo scorso; conseguentemente, nei paesi sviluppati vennero istituite commissioni che si occupavano della valutazione di tali effetti e dell'eventuale messa al bando dei prodotti più pericolosi. L'Italia in particolare regolamenta la messa in commercio dei presidi sanitari per la prima volta nel 1968: tutti presidi sanitari commercializzati nel territorio italiano devono essere autorizzati previa valutazione da parte di una commissione istituita presso il Ministero della Sanità della documentazione presentata dalla ditta produttrice su efficacia, caratteristiche chimiche fisiche tossicologiche, fitotossicità, spettro d'azione di tale sostanza, nonché le colture sulla quali va applicata. La disparità tra le procedure di registrazione seguite nei diversi paesi europei è stata superata a seguito dell'approvazione da parte dell'Unione Europea della famosa Direttiva 91/414, nella quale la difesa della salute del consumatore e la salubrità dell'ambiente venivano messe al primo posto. I nuovi criteri adottati nella direttiva 91/414 hanno determinato un processo di revisione relativo agli agrofarmaci commercializzati in Europa che ha comportato ad oggi la revoca del $67 \%$ delle sostanze attive presenti sul mercato al momento dell'adozione delle nuove procedure. La direttiva 91/414 ha inoltre armonizzato a livello europeo i cosiddetti LMR, limiti massimi di residui di agrofarmaci ammissibili nelle diverse derrate. L'opinione pubblica, molto preoccupata dalla presenza di tali residui, dovrebbe essere rassicurata dai risultati delle indagini effettuate dal Pesticide Action Network (PAN), su decine di migliaia di campioni prelevati in ben 29 stati europei: il $96 \%$ di tali campioni non conteneva residui individuabili con i metodi ufficialmente adottati per le analisi o ne conteneva in quantità inferiori al LMR. Per contro, mancano dettagliate informazioni sulla contaminazione delle derrate agricole e dei prodotti derivati dovuta alle micotossine, che presentano effetti tossici su uomo ed animali spesso peggiori rispetto a quelli determinati dai residui degli agrofarmaci; in ogni caso tali dati non sembrano suscitare particolare interesse nell'opinione pubblica, che ha anche poca o nulla considerazione per l'operatore agricolo, che durante lo svolgimento delle operazioni colturali è direttamente esposto all'agrofarmaco. Il 21 ottobre 2009 la Comunità Europea ha adottato due provvedimenti, il Regolamento 1107, che abroga e sostituisce la 91/414, e la Direttiva 128 che istituisce un quadro per l'azione comunitaria ai fini dell'utilizzo sostenibile degli agrofarmaci, indicati nella versione italiana con la pessima traduzione letterale pesticidi. Il Regolamento 1107 si propone di assicurare un elevato livello di protezione della salute umana e animale e dell'ambiente (...) attraverso l'armonizzazione delle norme relative all'immissione sul mercato dei prodotti fitosanitari, stimolando nel contempo la produzione agricola. Le disposizioni del Regolamento si fondano sul principio di precauzione al fine di garantire che le sostanze attive o i prodotti immessi sul mercato non abbiano effetti nocivi per la salute umana o animale o l'ambiente. A seguito dell'applicazione delle nuove normative, dal 20 al $60 \%$ delle sostanze attive che hanno superato la precedente revisione sono a rischio eliminazione, con possibili ripercussioni negative sulla protezione di colture minori e ridotta disponibilità di sostanze attive a diverso meccanismo d'azione utilizzabile per ridurre il rischio di selezionare ceppi resistenti. La Direttiva 128 sull'uso sostenibile degli agrofarmaci si propone di raggiungere il suo obiettivo in due modi fondamentali: l'informazione diretta agli operatori del settore e l'applicazione di strategie di protezione integrata (PI) su tutte le colture europee entro il 1 gennaio 2014. Lodevole è l'attenzione riservata dalla Direttiva all'operatore che viene tutelato mediante l'applicazione di misure protettive da adottare nell'esecuzione dei trattamenti. La PI è attualmente basata in Italia su protocolli predisposti dai Servizi Fitosanitari per le principali colture che agevoleranno sicuramente l'applicazione della Direttiva 128. Il ricorso a modelli matematici per la valutazione del rischio di infezione/infestazione, previsto dalla Direttiva, permetterà di conseguire un'ulteriore razionalizzazione della strategia di intervento e di utilizzare al meglio gli agrofarmaci disponibili.
Questo sarà possibile solo grazie all'impegno di tecnici preparati in diretto contatto con la realtà agricola che garantiscano agli agricoltori l'accesso alle informazioni necessarie per l'impostazione di un'efficace PI e ne promuovano una formazione permanente, nonché al potenziamento di strumenti quali reti di rilevamento agrometeorologico e di monitoraggio dell'andamento di infestazioni e malattie che rendono possibile la valutazione del rischio. L'adozione delle nuove disposizioni europee rappresenta una sfida per tutti coloro che si occupano di strategie di intervento ed in particolare per i ricercatori perché solo l'acquisizione di ulteriori approfondite conoscenze sugli agenti delle avversità delle piante permetterà di modellizzare in modo attendibile epidemie ed infestazioni. È importante che le acquisizioni in ambito scientifico vengano utilizzate per fornire indicazioni sul campo perché, come affermato da Russell, Presidente della British Society for Plant Pathology (2006), plant protection exists with the aim to secure food and other resources for the benefit of us all.

\section{Bibliografia}

Beddington J., 2010. Food security: contributions from science to a new and greener revolution. Phil. Trans. R. Soc. B 365:61-71.

Bentley J.W., Boa E., Danielsen S., Franco P., Antezana 0., Villarroel B., Rodriguez H., Ferrufino J., Franco J., Pereira R., Herbas J., Diaz 0., Lino V., Villarroel J., Almendras F., Colque S., 2009. Plant health clinics in Bolivia 2000-2009: operations and preliminary results. Food Sec. 1:371-386.

Conway G., 2007. A doubly green revolution: ecology and food production. In: R. May and A. McLean (eds.) Theoretical ecology: principles and applications. Oxford University Press, Oxford, UK, pp 158-171.

Evenson R. E., Gollin D., 2003. Assessing the impact of the green revolution from 1960 to 2000 . Science 300:758-762.

Flood J., 2010. The importance of plant health in food security. Food Sec. 2:215-231.

Fry W., 2008. Phytophthora infestans: the plant (and R gene) destroyer. Mol. Plant Pathol. 9:385-402.

Godfray C.H., Beddington J.R., Crute I.R., Haddad L., Lawrence D., Muir J.F., Pretty J., Robinson S., Thomas S.M., Toulmin C., 2010. Food security: the challenge of feeding 9 billion people. Science 327:812-818.

Njau P.N., Jin Y., Huerta-Espino J., Keller B., Singh R.P., 2010. Identification and evaluation of sources of resistance to stem rust race Ug99 in wheat. Plant Dis. 94:413-419.

Oerke E.C., 2006. Crop losses to pests. J. Agr. Sci. 144:31-43.

Pretorius Z.A., Singh R.P., Wagoire W.W., Payne T.S., 1999. Detection of virulence to wheat stem rust resistance gene Sr31 in Puccinia graminis f. sp. tritici in Uganda. Plant Dis. 84:203.

Russell P.E., 2006. The development of commercial disease control. Plant Pathol. 55:585-594.

Singh R.P., Hodson D.P., Jinz Y., Huerta-Espinos J., Kinyua M.G., Wanyera R., Njau P., Ward R.W., 2006. Current status, likely migration and strategies to mitigate the threat to wheat production from race Ug99 (TTKS) of stem rust pathogen. Cab Reviews: Perspectives in Agriculture, Veterinary, Nutrition and Natural Resources. CAB International United Kingdom. 54:1-13.

Strange R.N., Scott P.R., 2005. Plant disease: a threat to global food security. Annu. Rev. Phytopathol. 43:83-116.

Tisdale W.H., Williams I., 1934. Disinfectant and fungicide. US Patent 1972961. 